\title{
PROGRESS WITH THE PfSPZ MALARIA VACCINE
}

There has been substantial progress in the control of malaria during the past decade but it is estimated that in 2015 there were still 429,000 (uncertainty interval 235-639,000) deaths from malaria, mainly in African children, ${ }^{1}$ and current gains are threatened by the emergence of artemisinin and insecticide resistance. New tools, including malaria vaccines, are needed. The malaria vaccine RTS,S/AS01 has so far attracted the most attention, as it is the first malaria vaccine to obtain a positive approval from a regulatory authority ${ }^{2}$ following a long period of development and evaluation. ${ }^{3}$ However, during this period, substantial progress has been made with the development of several other malaria vaccines, including the Plasmodium falciparum sporozoite vaccine PfSPZ.

In this edition of Lancet Infectious Diseases Sissoko, Healy and colleagues report the results of one of the first efficacy trials of this vaccine undertaken in a malaria endemic country. ${ }^{4}$ The development of PfSPZ has been based on the observation made in the 1970s that immunisation with irradiated sporozoites, delivered through the bites of over 1,000 infected mosquitoes, provided protection against challenge from a mosquito infected with viable sporozoites. ${ }^{5}$ Irradiated sporozoites undergo partial development in liver cells to form a schizont, which induces an immune response, but which dies before rupturing into the blood stream and releasing the blood stage parasites which cause symptoms. Development of a vaccine based on irradiated sporozoites was for many years considered to be impractical because of the need for delivery by mosquito bite and a requirement to store the vaccine at a very low temperature. However, with courage and persistence, Hoffman and his colleagues at Sanaria have overcome these challenges by developing novel methods 
for producing purified sporozoite, and for storing and distributing them using a system based on liquid nitrogen. Many trials of PfSPZ have now been conducted in non-immune volunteers in order to define an optimum dose and route of administration. During a recent trial, $7 / 10$ American volunteers immunised intravenously five times with $2.7 \times 10^{5}$ sporozites were protected six months after vaccination against challenge with a homologous strain of parasite to that used in making the vaccine but only 1 of 10 challenged with a heterologous strain was protected, ${ }^{6}$ the kind of challenge likely to occur in malaria endemic populations. In the study reported by Sissoko and Healy, ${ }^{4} 46$ healthy adult Malians were vaccinated intravenously with five doses of $2.7 \times 10^{5}$ irradiated sporozoites and 47 controls received a placebo. The vaccine was safe and extremely well tolerated, producing minimal local or systemic side effects and only 1 of 502 intravenous injections had to be repeated. Efficacy was not as high as that seen in the American volunteers (48.3\% [95\% Cl 14.5, 68.7]) when measured against first infection and $28.8 \%$ [95\% $\mathrm{Cl} 8.2,47.2]$ against all infections during the five month follow-up period. The geometric mean titre of antibody to the circumsporozoite protein on the surface of the sporozoite, a partial correlate of protection, was substantially lower in the Malian volunteers than observed in American adults, suggesting that prior exposure to malaria may have down regulated the immune response to the malaria antigens delivered through the vaccine. ${ }^{7}$ Recent immunological studies have provided evidence to support this hypothesis with demonstration that malaria exposed individuals have an increased population of atypical memory B cells and a depletion of follicular helper T cells, which are important for development of immunological memory. ${ }^{8,9}$ Potential ways of overcoming this challenge for PfSPZ are administration of an increased dose and 
vaccination of children before they are exposed to malaria, approaches that are now being explored. ${ }^{10}$

Although most experience has been obtained with sporozoites attenuated by irradiation, parallel studies are being undertaken with sporozoites attenuated by genetic engineering ${ }^{11}$ or by administration of viable sporozoites under cover of administration of chloroquine. ${ }^{12}$ These approaches allow more complete development of the liver schizont than irradiated sporozoites and hence a broader immune response. Genetically modified sporozoite vaccines could be produced using the same manufacturing processing developed for PFSPZ. PfSPZ is a very promising malaria vaccine candidate which is likely to be deployed first in the military and travellers and perhaps in mass vaccination campaigns targeting elimination. Whether it can become part of the routine expanded programme of immunisation programme in highly malaria endemic countries, such as Mali, requires further research.

Word count: 710

Brian Greenwood

Faculty of Infectious and Tropical Diseases, London School of Hygiene \& Tropical Medicine, Keppel St., London WC1E 7HT, UK.

(e mail: brian.greenwood@Ishtm.ac.uk)

I declare no competing interests. 


\section{REFERENCES}

1. World Health Organization. World Malaria Report 2016. World Health Organization, Geneva, 2016.

2. European Medicines Agency (EMA). First malaria vaccine receives positive scientific opinion from EMA.

http://www.ema.europa.eu/ema/index.jsp?curl=pages/news and events/news/2015/0 7/news detail 002376.jsp\&mid=WC0b01ac058004d5c1

3. RTS,S Clinical trials Partnership. Efficacy and safety of RTS,S/ASO1 malaria vaccine with or without a booster dose in infants and children in Africa: final results of a phase 3, individually randomised, controlled trial. Lancet 2015; 386:31-45.

4. Sissoko MS, Healy SA, Katile A, et al. Safety and efficacy of PfSPZ vaccine via direct venous inoculation: a randomised, double blind study in healthy malaria exposed Malian adults. Lancet Infect Dis 2017; in press.

5. Clyde DF, Most H, McCarthy VC, Vanderberg JP. Immunization of man against sporozoite-induced falciparum malaria. Amer J Med Sci 1973; 266:169-77.

6. Epstein JE, Paolino KM, Richie TL, et al. Protection against Plasmodium falciparum malaria by PfSPZ vaccine. JCI Insight 2017; 2: e89154.

7. Illingworth J, Butler NS, Roetynck S, et al. Chronic exposure to Plasmodium falciparum is associated with phenotypic evidence of B and T cell exhaustion. J immunol 2013; 190; 1038-47.

8. Weiss GE, Crompton PD, Li S, et al. Atypical memory B cells are greatly expanded in individuals living in a malaria-endemic area. J immunol 2009; 183:2176-82. 
9. Ryg-Cornejo V, loannidas $L$, Ly A, et al. Severe malaria infections impair germinal center responses by inhibiting T follicular helper cell differentiation. Cell Reports 2016; 14; 6881.

10. Richie TL, Billingsley PF, Sim BK, et al Progress with Plasmodium falciparum sporozoites (PfSPZ)-based malaria vaccines. Vaccine 2015; 33:7452-61.

11. Kublin JG, Mikolajczak SA, Sack BK et al. Complete attenuation of genetically engineered Plasmodium falciparum sporozoites in human subjects. Sci Transl Med 2017; 9: eaad9099.

12. Roestenberg $M$, Teirlink AC, McCall MB, et al. Long-term protection against malaria after experimental sporozoite inoculation: an open-label follow-up study. Lancet 2011; 377:1770-76. 